\title{
Modified temporalis tendon transfer extended with periosteum for facial paralysis patients
}

\author{
Byeong Soo Kwon, \\ Hook Sun, \\ Jin Woo Kim \\ Department of Plastic Surgery, Inje \\ University Busan Paik Hospital, Busan, \\ Korea
}

\begin{abstract}
Background: We have devised a novel surgical method, termed as temporalis muscle tendonperiosteum (T-P) compound surgical method, by modifying pre-existing techniques. Our method is characterized by elevation of temporalis muscle tendon and the periosteum of the mandibular ramus as a single compound. Here, we describe the concept and clinical outcomes of our method. Methods: We conducted both a cadaveric study and a clinical study. First, we used four human cadavers (two males and two females) to confirm the anatomy of the temporalis muscle tendon and availability of sufficient length extension through the elevation of the T-P compound. Moreover, we obtained measurements of the mouth angle and the philtrum angle from a total of six patients (two males and four females) and compared them between preoperatively and postoperatively.

Results: The mean length of the periosteal portion was measured as $2.43 \pm 0.15 \mathrm{~cm}$ (range, 2.2$2.6 \mathrm{~cm})$. There was an improvement in the mouth angle postoperatively as compared with preoperatively $\left(7.2^{\circ} \pm 3.0^{\circ}\right.$ vs. $14.5^{\circ} \pm 4.7^{\circ}$, respectively). Moreover, there was also an improvement in the philtrum angle postoperatively as compared with preoperatively $\left(7.2^{\circ} \pm 3.4^{\circ} \mathrm{vs} .17 .2^{\circ} \pm 6.5^{\circ}\right.$, respectively).

Conclusion: Our method is a simple, minimally-invasive modality that is effective in achieving good clinical outcomes. Its advantages include an ability to achieve a firm extension of the temporalis muscle tendon as well as a lack of requirement for a donor site that may cause complications.
\end{abstract}

Keywords: Facial paralysis / Periosteum / Tendon transfer

\section{INTRODUCTION}

Diverse factors are involved in the pathogenesis of facial paralysis; these include congenital, traumatic, oncologic, neurologic, and iatrogenic ones. Once activated, the facial nerve has a great impact not only on the physical appearance but also on verbal and non-verbal communication. A cosmetic and functional recovery should be achieved in patients with facial paralysis. To date, many surgical treatment modalities, such as neurorrha-

\section{Correspondence: Jin Woo Kim}

Department of Plastic Surgery, Inje University Busan Paik Hospital, 75 Bokji-ro,

Busanjin-gu, Busan 47392, Korea

E-mail: jinooda@hanmail.net

Received October 6, 2020 / Revised December 14, 2020 / Accepted December 20, 2020 phy, nerve graft, muscle transplantation, and muscle transfer, have been attempted in such patients. Still, however, there are challenging problems in surgically treating them [1].

In 1934, Gillies [2] first described a novel surgical treatment modality for patients with facial paralysis by turning the temporalis muscle down over the zygomatic arch. But its disadvantages include bulging of the zygoma region caused by the temporalis muscle passing over the zygomatic arch, antidromic displacement of the temporalis muscle, overcorrection due to the short temporalis muscle tendon, and long incision scar left on the scalp after the elevation of the origin of the temporalis muscle. With technical advancements, various novel surgical modalities have been attempted [3-8]. These include orthodromic 
transfer of the temporalis muscle tendon, improving the method of antidromic turnover [9], a minimally-invasive transposition of the temporalis muscle tendon solely using the skin incision or oral incision $[5,10]$, and the transfer of the temporalis muscle tendon using a fascia lata graft. Of these, use of a fascia lata graft has been reported to be advantageous in lengthening the temporalis muscle tendon and correcting the philtrum deviation. But it may produce complications, such as seroma and donor-site infection, which poses a challenge for plastic surgeons $[3,4]$.

Given the above background, we have devised a novel surgical method, termed as "temporalis muscle tendon-periosteum ( $\mathrm{T}$ P) compound surgical method," by modifying pre-existing techniques. Our method is characterized by elevation of temporalis muscle tendon and the periosteum of the mandibular ramus as a single compound. It is effective in lengthening the temporalis muscle tendon without requiring a donor site that may produce complications. Here, we describe the concept and clinical outcomes of our method.

\section{METHODS}

\section{Cadaveric study}

For cadaveric study, we dissected one side of the face in four human cadavers (two males and two females). In each cadaver, we removed cheek skin, fat layer, superficial muscular aponeurotic system, and masseter muscle. This exposed the mandible and the temporalis muscle tendon. We ensured that the temporalis muscle tendon remained attached to the mandible. We made a periosteal incision from the junction between the tendon and bone to the retromolar triangle. Then, we performed a medial dissection of the subperiosteal layer from the area of the incision and isolated the periosteum from the overlying soft tissue. Subsequently, we dissected the periosteum at a width of 2 $\mathrm{cm}$. In the adjacent area to the coronoid process, we carefully dissected the subperiosteal layer while preserving the junction between the temporalis muscle tendon and periosteum. After dissecting the coronoid process using a reciprocating saw, we elevated the T-P compound. Following this, we carefully dissected the coronoid process from the T-P compound. This was followed by the measurement of the length of the periosteal portion in the T-P compound using a ruler for the purposes of estimating the length of possible extension (Fig. 1).

\section{Clinical study}

We performed a retrospective review of data obtained from a total of six patients (two males and four females) who had been treated at our medical institution between 2017 and 2019. In-

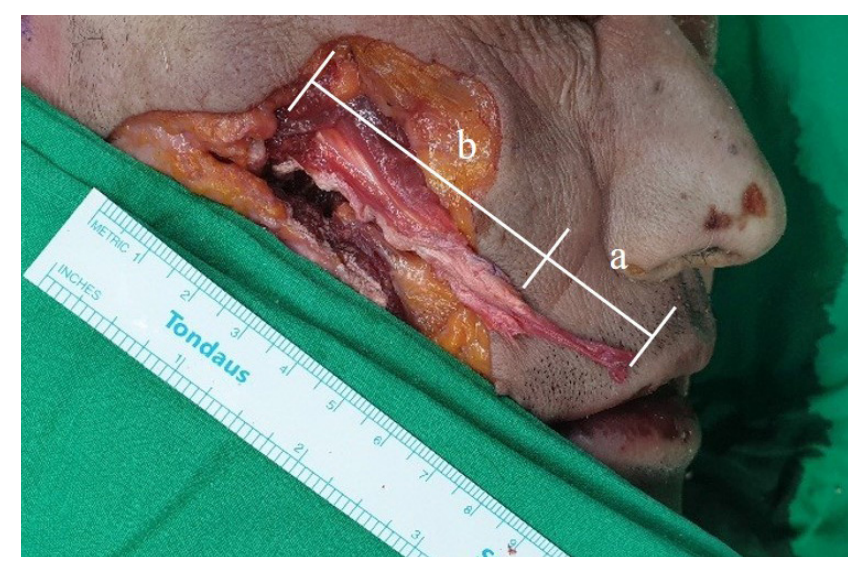

Fig. 1. Cadaveric study. The temporalis muscle tendon-periosteum (T-P) compound can be elevated through a firm connection between the temporalis muscle and the periosteum. a, periosteal portion of the T-P compound; $b$, temporalis muscle tendon portion of the T-P compound.

clusion criteria include a diagnosis of unilateral incomplete facial nerve palsy and a past history of undergoing our method. We also evaluated the patients who were in need of an overall correction of the facial asymmetry and then received subbrow lift, suprabrow lift, and blepharoptosis correction surgery concurrently with our method.

\section{Surgical technique}

Under general anesthesia, the entire facial area was preoperatively prepared, followed by irrigation of the oral cavity with povidone-iodine. The mouth was opened using a retractor and a vestibular incision was made accordingly. To prevent damages to the temporalis muscle tendon, an incision was solely made on the superficial layer. The masseter muscle, temporalis muscle, and buccinator muscles were exposed through a blunt dissection. Then, we examined whether the temporalis muscle tendon remained attached to the mandibular ramus. Subsequently, we made a downward incision to the depth of the periosteum from a slightly lateral part of the tendon attachment to the retromolar triangle. We performed a medial dissection of the subperiosteal layer from the area of the incision and thereby dissected the periosteum from the overlying soft tissue. Subsequently, we dissected the periosteum at a width of $2 \mathrm{~cm}$. Thus, we carefully performed a dissection along the subperiosteal layer towards the coronoid process up to 1-2 mm above the mandibular notch. The coronoid process was grasped using a Kelly forceps and then cut using a reciprocating saw. Using a Stryker drill, a hole was made on the fragment of the coronoid process. It was hung with a 2-0 nylon thread to prevent retraction.

A skin incision was parallelly made $0.2 \mathrm{~cm}$ medial to the nasolabial fold at a length of $0.2 \mathrm{~cm}$. Following this, we performed 
a blunt dissection to make a tunnel from the site of skin incision to that of oral incision using Metzenbaum scissors. Meanwhile, a probe was inserted in the Stensen's duct for protection. The T-P compound passed through the tunnel with a previously hung 2-0 nylon thread. At the site of skin incision, we performed a dissection above the orbicularis oris muscle. This was followed by appropriate fixation of the T-P compound with 3-0 Prolene sutures using a matrix suture method in the adjacent area to the mouth corner, for which the area $1-2 \mathrm{~cm}$ proximal to the end of the T-P compound served as the point of fixation. We preserved distal $1-2 \mathrm{~cm}$ of the compound to correct the deviation of the upper lip (Fig. 2).

A stab incision was made lateral 1/3 to the upper lip. A tunnel

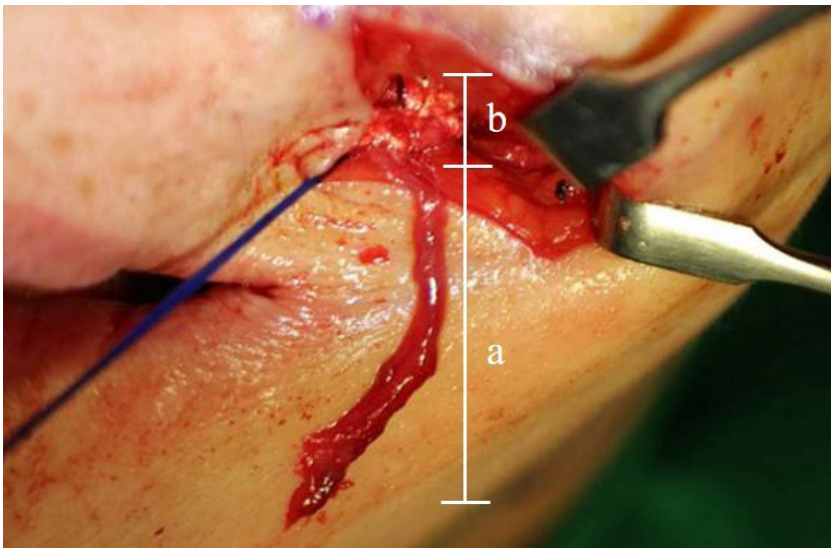

Fig. 2. Intraoperative photograph. The periosteal part of the remaining temporalis muscle tendon-periosteum (T-P) compound is exposed after its fixation to the mouth corner. a, periosteal portion of the T-P compound; $b$, temporalis muscle tendon portion of the T-P compound.

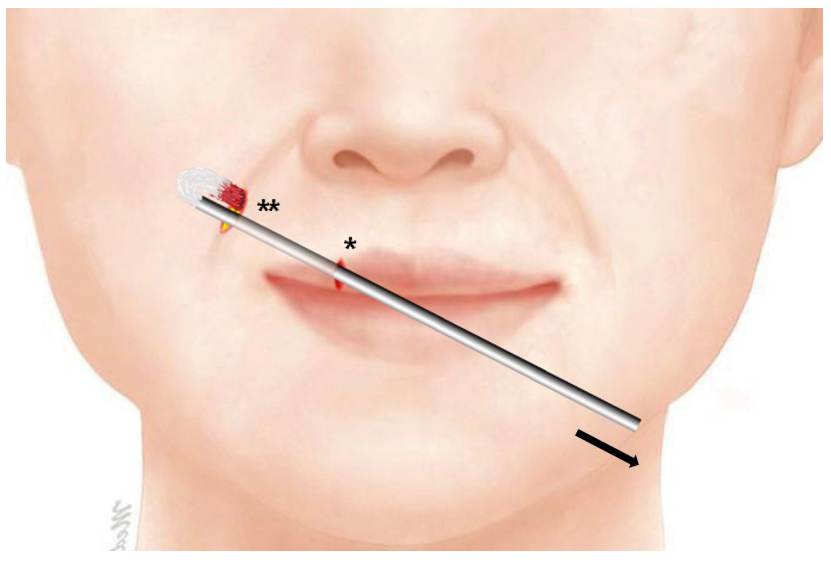

Fig. 3. Operative technique. The temporalis muscle tendon-periosteum (T-P) compound passes through the tunnel of upper lip by pulling out the catheter. The T-P compound is fixed to the orbicularis oris muscle, by which the upper lip deviation is corrected. ${ }^{*}$, a stab incision is made on the upper lip; ${ }^{* *}$, a skin incision is made in parallel to the nasolabial fold. was made by connecting between the site of skin incision and that of incision on the nasolabial fold using a liposuction catheter. The tip of the T-P compound was fixed to the tip of the liposuction catheter inserted in the tunnel with 6-0 Prolene sutures. By attracting the catheter towards the site of stab incision, the T-P compound passed through the upper lip tunnel and exited from the site of stab incision. The deviation of upper lip was corrected by fixing the T-P compound to the orbicular oris at the site of stab incision using 6-0 Prolene sutures (Fig. 3). Intraoral mucosa was sutured tightly with 4-0 Vicryl using an interlocking suture method, followed by a layer-by-layer suturing of the skin incision.

\section{Criteria for evaluating clinical outcomes}

Clinical outcomes were evaluated at 4-6 months postoperatively, for which postoperative measurements were obtained using a ruler and a protractor and then compared with preoperative ones based on both preoperative and postoperative photo-

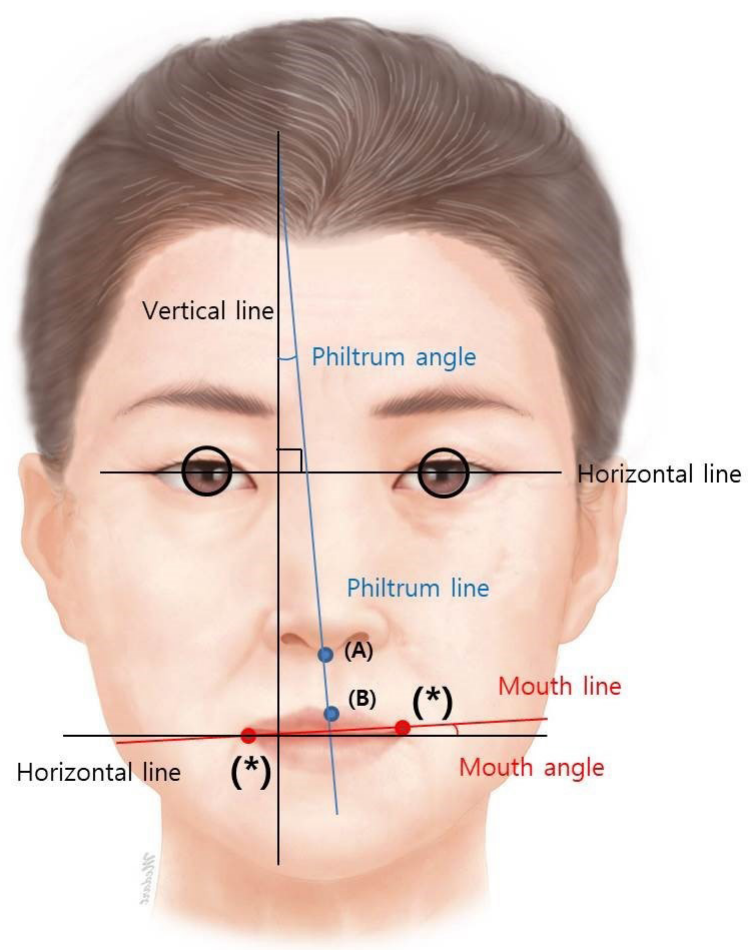

Fig. 4. Assessment of clinical outcomes. A line connecting between the centers of the pupils is defined as the horizontal line, and that perpendicular to the horizontal line is defined as the vertical line. The point of contact between the nasal columella and the philtrum is marked as (A) and the mid point of the cupid bow is marked as (B). A line connecting between (A) and (B) is defined as the philtrum line. Symbol $\left(^{*}\right)$ indicates the mouth corner. A line connecting between these symbols is defined as a straight line. The mouth angle refers to the angle formed between the horizontal line and the mouth line. The philtrum angle refers to the angle formed between the vertical line and the philtrum line. 
graphs.

Initially, an imaginary limbus margin was drawn in a circular shape over both eyes on the patient's photograph. A straight line connecting between the centers of the pupils was drawn, thus defined as "the horizontal line." Then, a straight line connecting between the patient's mouth corners was drawn, thus defined as "the mouth line." This was followed by the measurement of the angle formed between the horizontal line and the mouth line, serving as the mouth angle, for the purposes of assessing the degree of mouth deviation. Additionally, a straight line perpendicular to the horizontal line was drawn, thus defined as "the vertical line." A straight line connecting the point contact between the nasal columella and the philtrum (A in Fig. 4) and the midpoint of the cupid bow (B in Fig. 4) was drawn and defined as "the philtrum line." This was followed by the measurement of the angle formed between the vertical line and the philtrum line, serving as the philtrum angle, for the purposes of assessing the degree of philtrum deviation.

\section{RESULTS}

\section{Results of cadaveric study}

The mean length of the periosteal portion was measured as $2.43 \pm 0.15 \mathrm{~cm}$ (range, $2.2-2.6 \mathrm{~cm}$ ), as shown in Table 1 .

\section{Results of clinical study}

Baseline characteristics of the patients and clinical outcomes are summarized in Table 2. A total of six patients, comprising four men and two women, were evaluated; their mean age was

Table 1. Length of the periosteal portion

\begin{tabular}{lc}
\hline Length $(\mathrm{cm})$ & Value \\
\hline Mean \pm SD & $2.43 \pm 0.15$ \\
Cadaver 1 & 2.5 \\
Cadaver 2 & 2.2 \\
Cadaver 3 & 2.4 \\
Cadaver 4 & 2.6 \\
\hline
\end{tabular}

$61.0 \pm 6.2$ years (range, 52-69 years). Our clinical series of the patients include two cases $(33.3 \%)$ of congenital facial palsy, one case $(16.7 \%)$ of brain tumor-related facial paralysis, one case $(16.7 \%)$ of Bell's palsy, one case $(16.7 \%)$ of traumatic facial palsy, one case $(16.7 \%)$ of iatrogenic facial palsy, and one case (16.7\%) of stroke-related facial palsy.

There was an improvement in the mouth angle postoperatively as compared with preoperatively $\left(7.2^{\circ} \pm 3.0^{\circ}\right.$ vs. $14.5^{\circ} \pm 4.7^{\circ}$, respectively). Moreover, there was also an improvement in the philtrum angle postoperatively as compared with preoperatively $\left(7.2^{\circ} \pm 3.4^{\circ}\right.$ vs. $17.2^{\circ} \pm 6.5^{\circ}$, respectively). These results indicate that there were improvements in the mouth angle and the philtrum angle by $50 \%$ and $58 \%$, respectively, based on which it can be determined that our method was effective in improving the facial asymmetry.

All the patients underwent uneventful course with no postoperative complications.

\section{Illustrative cases}

Representative cases are presented in Figs. 5-7.
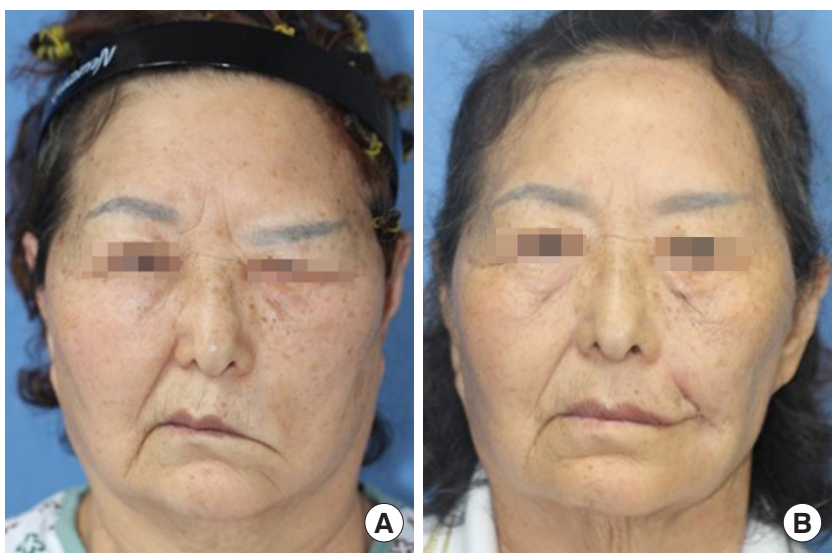

Fig. 5. A 69-year-old woman with congenital facial palsy. The patient underwent orthodromic transfer of the temporalis muscle using the temporalis muscle tendon-periosteum compound concurrently with subbrow lift for the left face. (A) Preoperative view. (B) Six-month postoperative view.

Table 2. Clinical outcomes

\begin{tabular}{|c|c|c|c|c|c|c|c|c|}
\hline \multirow{2}{*}{ No. } & \multirow{2}{*}{ Age (yr) } & \multirow{2}{*}{ Sex } & \multirow{2}{*}{ Causes } & \multicolumn{2}{|c|}{ Mouth angle $\left(^{\circ}\right)$} & \multicolumn{2}{|c|}{ Philtrum angle $\left({ }^{\circ}\right)$} & \multirow{2}{*}{ Complications } \\
\hline & & & & Preoperative & Postoperative & Preoperative & Postoperative & \\
\hline 1 & 69 & Female & Congenital & 18 & 7 & 25 & 7 & None \\
\hline 2 & 58 & Female & Bell's palsy & 15 & 10 & 10 & 5 & None \\
\hline 3 & 58 & Male & Traumatic & 21 & 11 & 25 & 12 & None \\
\hline 4 & 69 & Male & Brain tumor & 7 & $-5^{\text {a) }}$ & 10 & 2 & None \\
\hline 5 & 60 & Male & Congenital & 16 & 8 & 20 & 11 & None \\
\hline 6 & 52 & Male & Stroke & 19 & 2 & 13 & 6 & None \\
\hline
\end{tabular}

a) Negative value indicates an overcorrection, for which the absolute value was used. 

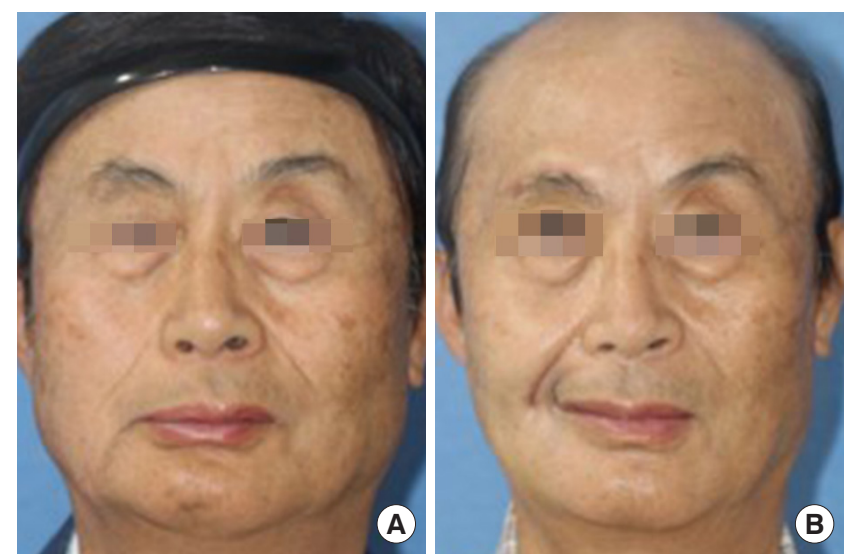

Fig. 6. A 69-year-old man with right hemifacial palsy. The patient underwent orthodromic transfer of the temporalis muscle using the temporalis muscle tendon-periosteum compound. (A) Preoperative view. (B) Five-month postoperative view.

\section{DISCUSSION}

Since the 1930s, various surgical methods have been developed to treat patients with facial palsy; these encompass the transfer of the temporalis muscle and the nerve graft [1]. The surgical modality introduced by Gillies in 1934 [2], where the temporalis muscle is dissected from the temporal bone and then turned over the zygomatic arch, revealed its disadvantages such as depression of the temporal area and midface widening. The orthodromic transfer of temporalis muscle was introduced by Mclaughlin in 1953, which is based on contraction of musclespecific vector [9]. Thus, it attempted to overcome disadvantages, such as midface widening and temporal area depression by transferring the temporalis muscle tendon to the mouth corner [9]. Subsequently, a method was developed by extending the length of the temporalis muscle tendon using fascia lata graft for the correction of mouth deviation by fixing the elongated temporalis muscle tendon in parallel to the lip [6]. Due to the requirement for a donor site, however, there is a possibility that donor site morbidity, including seroma and infection, might occur postoperatively $[3,4]$.

Our method is of significance in that we have developed it to overcome disadvantages of pre-existing methods. Of its several advantages, a lack of requirement for a donor site deserves special attention. According to Sidle and Fishman [3], donor site seroma occurred in patients undergoing orthodromic transfer of temporalis muscle tendon using fascia lata graft. Moreover, Pidgeon et al. [4] reported an occurrence of donor site infection. Requirement for a donor site may therefore raise the possibility of complications, such as donor site scar, pain, and prolonged operation time for harvesting the fascia lata. Another

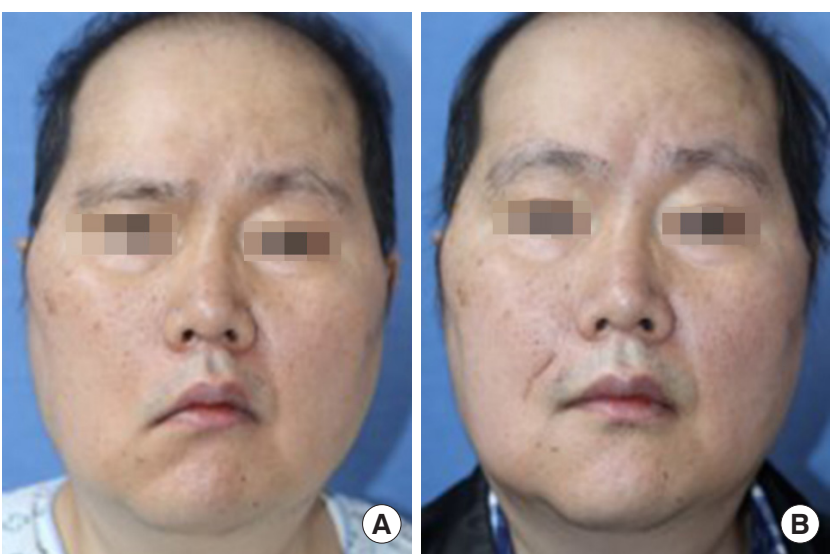

Fig. 7. A 52-year-old man with stroke-related facial palsy. The patient underwent orthodromic transfer of the temporalis muscle using the temporalis muscle tendon-periosteum compound concurrently with subbrow lift for the right face. (A) Preoperative view. (B) Four-month postoperative view.

advantage of our method is that we use the temporalis muscle tendon and periosteum as a single compound. The pre-existing fascia lata graft surgery involves suturing the fascia, which may cause cheese wiring at the junction or weakening of the junction $[4,6]$. By contrast, our method is effective in ensuring a more rigid junction. Moreover, our method is a minimally-invasive modality that leaves no visible scars, characterized by an incision covered by the nasolabial folds as well as an intraoral incision, which is well contrast to the pre-existing method based on a hemicoronal incision and a retromandibular incision $[1,5]$.

The temporalis muscle originates from the temporal line of the parietal bone, runs down the zygomatic arch and is attached to the coronoid process of the mandible. It plays a role in preventing the occurrence of temporomandibular dislocation, and also belongs to the muscles of mastication. Benninger and Lee [11] conducted a cadaveric study, thus reporting that the temporalis muscle tendon attached to the coronoid process can be divided into three parts: anterior distal muscle fiber, distal medial tendon head of temporalis, and distal lateral tendon head of temporalis. The anterior distal muscle fibers, commonly known as the temporalis muscle, were used in the current study; they were attached 1-2 cm below the coronoid process. According to Benninger and Lee [11], there were anatomical variations of the anterior distal muscle fibers attached below the anterior border of the ramus. It is therefore probable that the junction of the temporalis muscle tendon may be present at an unexpected location. That is, anatomical variations were considered as a critical factor in developing our method. In cases of damages to the anterior distal muscle fiber, the distal medial tendon head of the temporalis and the distal lateral tendon 
head of the temporalis may be alternatively used.

Fascia lata graft has been used for the orthodromic transfer of temporalis muscle tendon to reduce the tension on the tendon by extending the length of the temporalis muscle tendon. Correction of lip deviation can be performed with the remaining fascia. The fascia lata is a strong, extensible structure; it can be harvested at a length of $12-14 \mathrm{~cm}$ and a width of $1-2.5 \mathrm{~cm}[4,6]$. But we use the periosteum alternatively to the fascia lata. It can be harvested at a length of approximately $2.5 \mathrm{~cm}$ and a width of $2 \mathrm{~cm}$. Despite a shorter length and a smaller width of the periosteum, there were no complications due to the use of shorter T-P compound in our series. Presumably, this might be because the orthodromic transfer of temporalis muscle tendon transfer can be done without fascia lata graft $[5,10]$. It is also possible to extend the length even when the length is deemed insufficient. Therefore, we performed a blunt dissection towards the origin of the temporalis muscle and then isolated the coronoid process from the T-P compound. Moreover, we also considered a difference in the toughness between the periosteum and the fascia lata. We therefore obtain a greater strength of the T-P compound through a multilayered folding and overlapping. Thus, we could obtain a sufficient length and strength of the T-P compound.

Our results showed that both the philtrum angle and the mouth angle were also corrected to almost $0^{\circ}$. This indicates that our method is a clinically feasible modality. But we failed to assess its clinical outcomes as compared with pre-existing methods. This is one of the limitations of the current study.

In conclusion, our method is a simple, minimally-invasive modality that is effective in achieving good clinical outcomes. Its advantages include an ability to achieve a firm extension of the temporalis muscle tendon as well as a lack of requirement for a donor site that may cause complications.

\section{NOTES}

\section{Conflict of interest}

No potential conflict of interest relevant to this article was reported.

\section{Ethical approval}

The study was approved by the Institutional Review Board of Inje University Busan Paik Hospital (IRB No. 20-0176) and performed in accordance with the principles of the Declaration of Helsinki. Written informed consent was obtained.

\section{Patient consent}

The patients provided written informed consent for the publication and the use of their images.

\section{ORCID}

Byeong Soo Kwon https://orcid.org/0000-0002-7550-3994

Hook Sun https://orcid.org/0000-0003-0104-2598

Jin Woo Kim https://orcid.org/0000-0002-7660-843X

\section{REFERENCES}

1. Fattah A, Borschel GH, Manktelow RT, Bezuhly M, Zuker RM. Facial palsy and reconstruction. Plast Reconstr Surg 2012;129: 340e-352e.

2. Gillies H. Experiences with fascia lata grafts in the operative treatment of facial paralysis: (section of otology and section of laryngology). Proc R Soc Med 1934;27:1372-82.

3. Sidle DM, Fishman AJ. Modification of the orthodromic temporalis tendon transfer technique for reanimation of the paralyzed face. Otolaryngol Head Neck Surg 2011;145:18-23.

4. Pidgeon TE, Boca R, Fatah F. A technique for facial reanimation: the partial temporalis muscle-tendon transfer with a fascia lata sling. J Plast Reconstr Aesthet Surg 2017;70:313-21.

5. Boahene KD, Farrag TY, Ishii L, Byrne PJ. Minimally invasive temporalis tendon transposition. Arch Facial Plast Surg 2011; 13:8-13.

6. Aum JH, Kang DH, Oh SA, Gu JH. Orthodromic transfer of the temporalis muscle in incomplete facial nerve palsy. Arch Plast Surg 2013;40:348-52.

7. Sidle DM, Simon P. State of the art in treatment of facial paralysis with temporalis tendon transfer. Curr Opin Otolaryngol Head Neck Surg 2013;21:358-64.

8. Kim MJ, Oh TS. Treatment for ophthalmic paralysis: functional and aesthetic optimization. Arch Craniofac Surg 2019;20:3-9.

9. Mclaughlin CR. Surgical support in permanent facial paralysis. Plast Reconstr Surg (1946) 1953;11:302-14.

10. Leong SC, Lesser TH, Andrews P, Saeed SR. Transoral orthodromic temporalis muscle transfer technique for dynamic reanimation of the paralysed face. Clin Otolaryngol 2016;41:8313.

11. Benninger B, Lee BI. Clinical importance of morphology and nomenclature of distal attachment of temporalis tendon. J Oral Maxillofac Surg 2012;70:557-61. 